\title{
Endometriosis as Initial Manifestation of Myotonic Dystrophy Type-2
}

Josef Finsterer ${ }^{1, \odot} \quad$ Claudia Stöllberger $^{2}$

\footnotetext{
${ }^{1}$ Klinik Landstrasse, Messerli Institute, Vienna, Austria

${ }^{2}$ 2nd Medical Department with Cardiology and Intensive Care Medicine, Krankenanstalt Rudolfstiftung, Vienna, Austria
}

J Neurosci Rural Pract:2021;12:219-221

Though gynecological involvement (ovarian cysts, endometriosis, hypogonadism) has been occasionally reported in myotonic dystrophy type-2 (DM2), ${ }^{1,2}$ endometriosis as initial manifestation is unknown.

The patient is a 67-year-old female referred for nonsystemic vertigo and recurrent collapses for the last 10 months together with stocking-type sensory disturbances for the last 2 years and tingling of the toes bilaterally for the last 1 week. Her history was noteworthy for: endometriosis with severe abdominal pain 1 week prior to and 1 week postmenstruation since age 18 years requiring left ovariectomy at age 24 years; endometriosis of the colon with stenosis of the sigma requiring resection of the sigma at age 30 years leading to resolution of perimenstrual abdominal pain; volvulus at age 30 years requiring surgery; recurrent surgery for hernia cicatrica at ages 31,32 , and 34 years; steatosis hepatis upon liver puncture at age 43 years; hysterectomy, right ovariectomy, and urinary bladder lifting at age 50 years; diabetes since age 53 years; right cataract-surgery at age 54 years; laser therapy at age 55 years; hyperlipidemia since age 55 years; myotonic and pseudomyotonic discharges at most investigated sites on needle electromyography from the anterior tibial muscle; inclusion body myopathy or DM2 (hypertrophic fibers, some atrophic fibers, rimmed and autophagic vacuoles, internalized nuclei) upon muscle biopsy because of hyper-CKemia and muscle weakness triggered by statins at age 56 years; cholecystectomy at age 57 years; hypoacusis since age 61 years; left cataract-surgery at age 64 years; chronic constipation since age 65 years; arterial hypertension since age 65 years; and recurrent collapses during exercise since age 67 years.
Address for correspondence Josef Finsterer, MD, PhD, Postfach 20, 1180 Vienna, Austria (e-mail: fifigs1@ yahoo.de).

Clinical neurologic exam at age 67 years revealed short stature, hypoacusis bilaterally (hearing devices), unrounded pupils, massively sore neck muscles, diffuse muscle weakness of upper limbs (MRC4-), diffuse wasting, positive pyramidal signs on the right side, weakness for right hip flexion (MRC5-), weakness for foot extension bilaterally (MRC5-), diffuse wasting, a subclonic left patella tendon reflex, reduced Achilles tendon reflexes, and hypoesthesia of all toes. There was a pes equino-excavatus. The Gower sign was positive. Creatine-kinase was normal. Cerebral magnetic resonance imaging revealed contact between the cerebelli anterior inferior artery and the vestibulo-cochlear nerves and nonspecific gliotic spots bilaterally. Cerebral computed tomography showed hyperostosis frontalis. Echocardiography revealed concentric thickening of the left-ventricular myocardium. Electrocardiogram was normal. On the Mini-Mental State Exam, she scored 27/30 points. Nerve conduction studies revealed axonal neuropathy of lower limbs: Ultrasonography revealed carpal tunnel syndrome bilaterally. Genetic work-up revealed a CCTG-repeat expansion of $>300 \mathrm{bp}$ ( $>75$ repeats) in ZNF9.

The presented patient is interesting for DM2 and endometriosis as initial manifestation of DM2. The diagnosis DM2 relied on the clinical presentation, electromyography, and genetics. The diagnosis was challenged by previously undescribed phenotypic features, such as occasionally mildly elevated serum lactate, short stature, and hyperostosis frontalis (-Table 1). Arguments for a causal relation between DM2 and endometriosis are that gynecological involvement has been previously reported in DM2 (-Table 1), ${ }^{3}$ that endocrine involvement is frequent in
DOI https://doi.org/ $10.1055 / \mathrm{s}-0040-1721545$ ISSN 0976-3147. (c) 2021. Association for Helping Neurosurgical Sick People.

This is an open access article published by Thieme under the terms of the Creative Commons Attribution-NonDerivative-NonCommercial-License, permitting copying and reproduction so long as the original work is given appropriate credit. Contents may not be used for commercial purposes, or adapted, remixed, transformed or built upon. (https://creativecommons.org/licenses/by-nc-nd/4.0/)

Thieme Medical and Scientific Publishers Pvt. Ltd. A-12, 2nd Floor, Sector 2, Noida-201301 UP, India 
Table 1 Clinical manifestations of DM1 and DM2 in the presented case and the literature

\begin{tabular}{|c|c|c|c|}
\hline Manifestation & Index case & DM1 & DM2 \\
\hline \multicolumn{4}{|l|}{ Muscle } \\
\hline Myopathic face & No & Yes & Yes \\
\hline Limb muscle weakness & Yes & Yes & Yes \\
\hline Clinical myotonia & No & Yes & Yes \\
\hline Myalgias & No & No & Yes \\
\hline Muscle wasting & Yes & Yes & Yes \\
\hline Calf hypertrophy & No & No & Yes \\
\hline Hyper-CKemia & Yes & Yes & Yes \\
\hline \multicolumn{4}{|l|}{ Peripheral nerves } \\
\hline Polyneuropathy & Yes & Yes & Yes \\
\hline Autonomic dysfunction & No & Yes & No \\
\hline Carpal tunnel syndrome & No & Yes & No \\
\hline \multicolumn{4}{|l|}{ Brain } \\
\hline Cognitive impairment & Yes & Yes & Yes \\
\hline Behavioral abnormalities & No & Yes & No \\
\hline Mental retardation & No & Yes & No \\
\hline Daytime sleepiness & No & Yes & Yes \\
\hline White matter lesions & Yes & Yes & Yes \\
\hline \multicolumn{4}{|l|}{ Eyes } \\
\hline Cataract & Yes & Yes & Yes \\
\hline Low intraocular pressure & No & Yes & No \\
\hline Pigmentary retinopathy & No & Yes & Yes \\
\hline Epiretinal membranes & No & Yes & Yes \\
\hline \multicolumn{4}{|l|}{ Endocrine } \\
\hline Diabetes & Yes & Yes & Yes \\
\hline Hyperthyroidism & No & No & Yes \\
\hline Hypothyroidism & No & Yes & \\
\hline Hyperhidrosis & No & No & Yes \\
\hline Hypogonadism & No & Yes & Yes \\
\hline Hyperparathyroidism & No & Yes & No \\
\hline Abortus, stillbirth & No & Yes & No \\
\hline Endometriosis & Yes & Yes & Yes \\
\hline Osteoporosis & No & Yes & No \\
\hline Short stature & Yes & No & No \\
\hline \multicolumn{4}{|l|}{ Cardiac } \\
\hline Conduction defects & Yes & Yes & Yes \\
\hline Arterial hypertension & Yes & Yes & Yes \\
\hline Myocardial thickening & Yes & Yes & Yes \\
\hline Dilative cardiomyopathy & No & Yes & Yes \\
\hline Noncompaction & No & Yes & Yes \\
\hline
\end{tabular}

DM2, that endometriosis has been reported in DM1/DM2 ( - Table 1), ${ }^{4,5}$ and that no other first-degree relatives had endometriosis.
Overall, the phenotypic spectrum of DM2 is broader than anticipated and resembles DM1 in many aspects. 
Table 1 (Continued)

\begin{tabular}{|c|c|c|c|}
\hline Manifestation & Index case & DM1 & DM2 \\
\hline \multicolumn{4}{|l|}{ Gastrointestinal } \\
\hline Dysphagia & No & Yes & Yes \\
\hline Dysmotility & Yes & Yes & Yes \\
\hline Steatosis hepatis & Yes & Yes & Yes \\
\hline Liver cirrhosis & No & No & Yes \\
\hline Elevated GGT & Yes & Yes & Yes \\
\hline Cholecystolithiasis & Yes & Yes & Yes \\
\hline Nonalcoholic fatty liver disease & Yes & Yes & Yes \\
\hline \multicolumn{4}{|l|}{ Bones } \\
\hline Hyperostosis frontalis & Yes & Yes & No \\
\hline Foot deformities & Yes & Yes & Yes \\
\hline Small sella & No & Yes & No \\
\hline Large air sinuses & No & Yes & No \\
\hline \multicolumn{4}{|l|}{ Skin } \\
\hline Frontal balding & No & Yes & Yes \\
\hline Pilomatricoma & No & Yes & No \\
\hline \multicolumn{4}{|l|}{ Others } \\
\hline Hydroureter & No & No & Yes \\
\hline Hyperlipidemia & Yes & Yes & Yes \\
\hline Hyperuricemia & Yes & No & Yes \\
\hline Thrombocytosis & No & No & Yes \\
\hline Renal cysts & No & No & Yes \\
\hline Low IgG, IgM & No & Yes & Yes \\
\hline Lactic acidosis & Yes & No & No \\
\hline
\end{tabular}

Abbreviations: DM1, myotonic dystrophy type-1; DM2, myotonic dystrophy type-2; GGT, gamma-glutamyl transferase; IgG, immunoglobulin G; IgM, immunoglobulin M.

\section{Ethical Approval}

Informed consent was obtained from the reported patient.

\section{Authors' Contributions}

Both authors contributed equally (J. F.: clinical investigations, design, literature search, discussion, first draft; C. S.: clinical investigations, literature search, discussion, critical comments).

\section{Funding}

None.

\section{Conflict of Interest}

None declared.

\section{References}

1 Finsterer J, Stöllberger C, Reining-Festa A, Loewe-Grgurin M, Gencik M. Myotonic dystrophy-2: unusual phenotype due to a small CCTG-expansion. Balkan J Med Genet 2018;21(2):39-43
2 Meola G, Moxley RT III. Myotonic dystrophy type 2 and related myotonic disorders. J Neurol 2004;251(10):1173-1182

3 Atlas I, Smolin A. Combined maternal and congenital myotonic dystrophy managed by a multidisciplinary team. Eur J Obstet Gynecol Reprod Biol 1999;87(2):175-178

4 Ota K, Nakamura Y, Nakamura E, et al. Massive abscess with prolonged respiratory failure due to newly diagnosed myotonic dystrophy: a case report. Medicine (Baltimore) 2019;98(17):e15427

5 Schoser B, Montagnese F, Bassez G, et al; Myotonic Dystrophy Foundation. Consensus-based care recommendations for adults with myotonic dystrophy type 2. Neurol Clin Pract 2019;9(4):343-353 